\section{Lamotrigine for agitation in older patients with dementia}

Although not approved by the Food and Drug Administration (FDA) for behavioral disturbances, the largest number of antipsychotic prescriptions in older adults is for this indication associated with dementia (Sultzer et al., 2008). In 2005, the FDA determined that atypical antipsychotic medications were associated with a 1.6 to 1.7 greater risk of mortality compared with placebo when administered to older patients with dementia (De-Deyn et al., 2005). Psychosis and agitation symptoms in patients with dementia are common, disruptive, and costly but there are no wellestablished, evidenced-based effective treatment alternatives (Jeste et al., (2008). A limited amount of mostly anecdotal scientific data suggests that anticonvulsant medications, including some of the newer agents like lamotrigine, may be helpful for agitation and psychosis in older patients with dementia (DeLeon, 2004). Lamotrigine is an antiepileptic drug whose mechanism of action is thought to be linked to voltage-sensitive sodium channel blockade in the neuronal membrane and inhibition of presynaptic glutamate and aspartate release (Baumann et al., 2007). Lamotrigine has a safe profile and has documented efficacy in delaying recurrence of mood episodes in patients with bipolar disorder in trials conducted for up to 18 months.

Our center conducted a retrospective medical record review study of the effectiveness (efficacy and safety) of lamotrigine for manic-like symptoms and agitation in older patients with dementia who were residing in assisted living facilities. The study was approved by the University of California, San Diego Human Research Protections Program, an Institutional Review Board. The diagnosis of dementia was based on DSM-IV criteria for Dementia of the Alzheimer's Type. The clinician's decision to start lamotrigine was based on either the presence of prominent manic-like symptoms or the failure to respond at tolerable dosages of an atypical antipsychotic medication. Patients were prescribed lamotrigine according to the usual titration recommended for adults. Unfortunately, the rating scales - Mini-mental State Examination (MMSE), Hamilton Depression Rating Scale (HAM-D) and Young Mania Rating Scale (YMRS) - were not used uniformly. Only patients who had completed scales on at least $80 \%$ of their visits were included in the study $(n=5)$. $\chi^{2}$, t test and ANOVA were used to analyze the data.

The patients in the study comprised two men and three women. Their average age was 77 years $(S D=9.2)$. Patients received doses of lamotrigine between $100 \mathrm{mg}$ and $300 \mathrm{mg}$ per day, with the average daily dose being $190 \mathrm{mg}$ per day. An analysis of change on the different rating scales through five months of treatment revealed that there was a non significant decrease of the MMSE from 19.6 to 18.2 , a non significant decrease of the HAM-D scale of 12.8 to 9 , and a significant decrease $(p=0.046)$ in the YMRS from 10.4 to 5 . See Figure S1, available as supplementary material attached to the electronic version of this letter at www.journals.cambridge.org/jid_IPG. None of the patients had to discontinue lamotrigine due to side effects.

In summary, the patients in this small study demonstrated improvement in mean YMRS score but had no change in mean total HAM-D score. None of the patients in this review had to discontinue lamotrigine due to side effects, suggesting a safe profile in this population (Baumann et al., 2007). In addition, none experienced a significant worsening in cognitive function as measured by MMSE. Our findings are preliminary and should be viewed with caution due to the very small sample size and retrospective nature of this study. Nonetheless, we believe our study provides some justification for a prospective study of lamotrigine for the treatment of agitation and psychosis in older patients with dementia in order to determine if it may truly be a safer and more effective alternative to the atypical antipsychotics.

\section{References}

Baumann, R., Fakhoury, T., Kustra, R., Vuong, A., Hammer, A. and Messenheimer, J. A. (2007). Conversion to lamotrigine monotherapy from valproate monotherapy in older adolescent patients with epilepsy. Current Medical Research and Opinion, 23, 24612465.

De-Deyn, P. P., Katz, I. R., Brodaty, H., Lyons, B., Greenspan, A. and Burns, A. (2005). Management of agitation, aggression and psychosis associated with dementia: a pooled analysis including three randomized, placebo-controlled double-blind trials in nursing home residents with risperidone. Clinical Neurology and Neurosurgery, 107, 497-508.

DeLeon, O. A. (2004). Treatment of psychotic symptoms with lamotrigine in Alzheimer's disease. Fournal of Clinical Psychopharmacology, 24, 232-233. 
Jeste, D. et al. (2008). ACNP White Paper: update on use of antipsychotic drugs in elderly persons with dementia. Neuropsychopharmacology, 33, 957-970.

Sultzer, D. et al. (2008). Clinical symptom responses to atypical antipsychotic medications in Alzheimer's disease. Phase 1: Outcomes from the CATIE-AD Effectiveness Trial. American fournal of Psychiatry [e-published ahead of print].
Bernardo NG, ${ }^{1}$ Alvaro CamaCho, ${ }^{2}$ WAYNE BARDWELL ${ }^{3}$ AND DANIEL D. SEWELL ${ }^{4}$

${ }^{1}$ Medical Director, Sun Valley Behavioral Medical Center, and Assistant Clinical Professor of Psychiatry. University of California, San Diego, U.S.A. Email: bng@sunvalleyb.com

${ }^{2}$ Medical Director. Imperial County Behavioral Health, and Assistant Clinical Professor of Psychiatry. University of California, San Diego, U.S.A.

${ }^{3}$ Director, Patient \& Family Support Services, La Jolla, California, U.S.A.

${ }^{4}$ Clinical Professor of Psychiatry. University of California, San Diego, U.S.A. 